\title{
Predictive learn and apply: MAVIS application - apply
}

Cranney, Jesse, Zhang, Hao, Doucet, Nicolas, Rigaut, François, Gratadour, Damien, et al.

Jesse Cranney, Hao Zhang, Nicolas Doucet, François Rigaut, Damien Gratadour, Visa Korkiakoski, José De Doná, Yuxi Hong, Hatem Ltaief, David Keyes, "Predictive learn and apply: MAVIS application - apply," Proc. SPIE 11448, Adaptive Optics Systems VII, 114482L (13 December 2020); doi: 10.1117/12.2561914

SPIE. Event: SPIE Astronomical Telescopes + Instrumentation, 2020, Online Only 


\title{
Predictive Learn and Apply: MAVIS Application - Apply
}

\author{
Jesse Cranney ${ }^{\mathrm{a}, \mathrm{b}}$, Hao Zhang ${ }^{\mathrm{a}}$, Nicolas Doucet ${ }^{\mathrm{a}}$, François Rigaut ${ }^{\mathrm{a}}$, Damien Gratadour ${ }^{\mathrm{a}, \mathrm{c}}$, \\ Visa Korkiakoski ${ }^{\mathrm{a}}$, José De Donáb ${ }^{\mathrm{b}}$ Yuxi Hong ${ }^{\mathrm{d}}$, Hatem Ltaief ${ }^{\mathrm{d}}$, and David Keyes ${ }^{\mathrm{d}}$

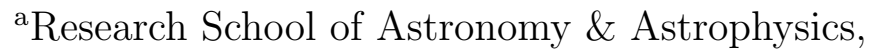 \\ College of Science, \\ Australian National University, Australia \\ bchool of Electrical Engineering \& Computing, \\ Faculty of Engineering and Built Environment, \\ University of Newcastle, Australia \\ ${ }^{\mathrm{c}}$ LESIA, Observatoire de Paris, \\ Université PSL, Sorbonne Université, Université de Paris, Sorbonne Paris Cité, \\ CNRS France \\ ${ }^{\mathrm{d}}$ Division of Computer, Electrical, and Mathematical Sciences and Engineering, \\ Extreme Computing Research Center, \\ King Abdullah University of Science and Technology, KSA
}

\begin{abstract}
The Learn \& Apply tomographic reconstructor coupled with the pseudo open-loop control scheme shows promising results in simulation for multi-conjugate adaptive optics systems. We motivate, derive, and demonstrate the inclusion of a predictive step in the Learn \& Apply tomographic reconstructor based on frozen-flow turbulence assumption. The addition of this predictive step provides an additional gain in performance, especially at larger wave-front sensor exposure periods, with no increase of online computational burden. We provide results using end-to-end numerical simulations for a multi-conjugate adaptive optics system for an $8 \mathrm{~m}$ telescope based on the MAVIS system design.
\end{abstract}

Keywords: Adaptive Optics, Predictive Control, MAVIS, MCAO, Learn and Apply, POLC.

\section{INTRODUCTION}

Adaptive Optics (AO) is crucial for obtaining diffraction limited performance in modern optical telescope systems. Classical single-conjugate AO (SCAO) allows for the correction of atmospheric turbulence in the direction of a given guide-star/wave-front sensor (WFS) pair. In general, the scientific target does not coincide with the guidestar, so a tomographic AO system with multiple WFSs (using independent guide-stars) can be implemented to reconstruct the atmospheric turbulence in real-time in the direction of the scientific target and project this turbulence to a deformable mirror (DM) at the telescope pupil in the direction of the scientific target, e.g., Laser-tomographic AO (LTAO). More generally, there may be a number of scientific targets, multiple DMs in closed-loop, and multiple WFSs, all of which combined constitute a so-called multi-conjugate AO (MCAO) system.

The real-time process of taking measurements from various WFSs and computing the commands to be applied to multiple DMs is commonly referred to as tomographic reconstruction, and is the subject of many studies in AO over the last two decades (see, e.g., 1-8). Here we discuss the Learn and Apply (L\&A) method for generating the tomographic reconstructor for general MCAO systems, and the extension of the classical L\&A method to include a predictive estimation based on the frozen-flow hypothesis in an attempt to reduce the servo-lag error introduced by inherent time-delays in AO systems. This predictive step depends on the knowledge of additional turbulence parameters, and in the spirit of the L\&A scheme, we provide a robust and efficient method of identifying these parameters (a.k.a., the learn-step) from AO telemetry in our companion paper by Zhang et al. ${ }^{9}$

Send correspondence to Jesse Cranney, E-mail: jesse.cranney@anu.edu.au

Adaptive Optics Systems VII, edited by Laura Schreiber, Dirk Schmidt, Elise Vernet, Proc. of SPIE

Vol. 11448, 114482L · (C) 2020 SPIE · CCC code: 0277-786X/20/\$21 · doi: 10.1117/12.2561914 


\subsection{Learn and Apply in AO}

The Learn and Apply ${ }^{1}$ (L\&A) method for generating a tomographic reconstructor in adaptive optics (AO) is a type of minimum mean-square error (MMSE) optimisation, based on heuristic principles. Despite its intuitive

formulation, the L\&A reconstructor embedded into the pseudo open-loop controller ${ }^{10,11}$ (POLC) is capable of providing high-performance correction in complex AO systems.

L\&A is based on the principle that finding a control scheme to optimise deformable mirror (DM) commands for correction of atmospheric turbulence in the direction of given wave-front sensors (WFSs) is straight-forward (e.g., the pseudo-inverse of the interaction matrix between DMs and WFSs), yet generally the WFS directions are not equivalent to the direction of the scientific targets. In L\&A, this is reconciled by using the geometry of the $\mathrm{AO}$ system and a model of the atmospheric profile to estimate artificial measurements in the direction(s) of the scientific target(s), based on the WFS measurements.

While this method is technically very similar to the traditional phase-based MMSE reconstructor (see, e.g., 3,4), there are several key differences which make L\&A interesting in its own right. From an optimisation perspective, the L\&A method is equivalent to regulating the residual slopes from the synthetic WFSs, rather than the residual wave-front phase. This difference appears to have practical advantages, such as the inclusion of non-common-path aberrations directly in the measurement space, and the opportunity to build a more data-driven turbulence model (though we do not investigate those here).

Another notable difference between L\&A and traditional MMSE is the dependency of a meta-pupil support. L\&A circumvents the need for defining an intermediate meta-pupil support (as can be seen in the upcoming equations). A more general reconstruction technique was proposed by Correia et al. ${ }^{7}$ - the so-called spatioangular (SA) reconstructor - which also avoids such a support. As noted in that article, L\&A turns out to be a special case of this SA reconstructor, and so the extension of L\&A to a predictive reconstructor (we refer to as pL\&A) is implied by Correia et al., albeit in phase-space and for an MOAO system. The purpose of this paper, as stated, is to demonstrate pL\&A in an MCAO system (nominally MAVIS ${ }^{12}$ ), but this could also be seen as a demonstration of a slope-based SA reconstructor for MCAO. A performance analysis of traditional MMSE in the context of MAVIS has been performed by Agapito et al. ${ }^{13}$

\subsection{L\&A Reconstructor}

The L\&A scheme is well suited for implementation in open-loop, and hence also POLC, whereby the linearity of the DMs and WFSs are exploited to reconstruct the effective open-loop measurements from each WFS. As such, it is appropriate to discuss the L\&A scheme as per its implementation in an open-loop controller, and then to use the POLC method to embed this estimator in a closed-loop system.

The main goal of L\&A, as stated above, is to estimate WFS measurements in the directions of targets obtained at time $k$ (which we denote $z_{k}$, and their estimate $\hat{z}_{k}$ ) from the actual measurements obtained at time $k$ (which we will denote $s_{k}$ ). All of these measurements are assumed to be open-loop (i.e., the DM does not affect the measurements). In classical L\&A, these are typically obtained using the linear least mean square error (LLMSE) estimate (see, e.g., 14) of $z_{k}$ given $s_{k}$ :

$$
\hat{z}_{k}=\left\langle z_{k} s_{k}^{\top}\right\rangle\left\langle s_{k} s_{k}^{\top}\right\rangle^{-1} s_{k},
$$

where $\langle\cdot\rangle$ denotes the expectation operator, and $s_{k}^{\top}$ denotes the transpose of $s_{k}$.

Once the estimate of $z_{k}$ is determined, the computation of the DM command is simply the solution to the regularised least-squares problem:

$$
\begin{aligned}
u_{k} & =\underset{u}{\arg \min }\left\|D_{z} u-\hat{z}_{k}\right\|^{2}+\alpha\|u\|^{2} \\
& =\underbrace{\left(D_{z}^{\top} D_{z}+\alpha \mathbf{I}\right) D_{z}^{\top}}_{C_{z}} \hat{z}_{k},
\end{aligned}
$$

where $D_{z}$ and $C_{z}$ are the interaction and control matrices (respectively) with respect to the synthetic WFSs. Combining Eqns. (1) \& (2) gives an expression for the L\&A reconstructor:

$$
u_{k}=\underbrace{C_{z}\left\langle z_{k} s_{k}^{\top}\right\rangle\left\langle s_{k} s_{k}^{\top}\right\rangle^{-1}}_{R} s_{k} .
$$


The two covariance matrices $\left(\left\langle z_{k} s_{k}^{\top}\right\rangle\right.$ and $\left.\left\langle s_{k} s_{k}^{\top}\right\rangle\right)$ in the L\&A scheme are updated regularly to account for variations in system parameters (denoted collectively by $\rho$ ), namely the atmospheric turbulence profile. The process of computing these matrices depends on identifying the unknown system parameters in the so-called learn-step, by performing a nonlinear regression on the recent telemetry. This optimisation task is classically performed using the Levenberg-Marquardt (LM) method (see, e.g., Vidal et al. ${ }^{1}$ )- a second order nonlinear least-squares optimiser. A recently published stochastic modification of LM, the so-called Stochastic LevenbergMarquardt (SLM) method by Hong et al. ${ }^{15}$ provides a GPU optimised implementation, showing a significant speedup in time-to-solution for VLT scale MCAO systems (e.g., MAVIS ${ }^{12}$ ) and beyond. Our companion paper by Zhang et al. ${ }^{9}$ discusses the performance (both numerical and time-to-solution) of the SLM method in a MAVIS-like system.

The output of the learn-step is the set of system parameters $\rho$ based on the most recent telemetry. These parameters are the input to the so-called apply-step, where the required covariance matrices $\left(\left\langle z_{k} s_{k}^{\top}\right\rangle\right.$ and $\left.\left\langle s_{k} s_{k}^{\top}\right\rangle\right)$ are populated, and the overall tomographic reconstructor is determined. The population of those matrices in the classical L\&A scheme is given in the following section, and the corresponding pL\&A matrices are given in Section 2.

\subsection{Populating the Covariance Matrices}

To populate the covariance matrices analytically, a model of the WFS geometry and turbulence structure function (e.g., von Kármán ${ }^{16}$ ) is required. In the measurement space, these models can be found in Gendron et al., ${ }^{17}$ and provide expressions for populating a general measurement-based covariance matrix $\left\langle z_{k} s_{k}^{\top}\right\rangle$ in the form of:

$$
\left\langle z_{k} s_{k}^{\top}\right\rangle_{i j}=\sum_{\ell=1}^{L} C_{\ell}\left(x_{i}^{\ell}, y_{i}^{\ell}, x_{j}^{\ell}, y_{j}^{\ell}, \rho\right),
$$

where $\left\langle z_{k} s_{k}^{\top}\right\rangle_{i j}$ is the $(i, j)^{\text {th }}$ element of $\left\langle z_{k} s_{k}^{\top}\right\rangle, L$ is the number of layers of atmosphere included in the model, and $C_{\ell}$ is the covariance function of the $\ell^{\text {th }}$ layer which is a function of the $(x, y)$ sub-aperture coordinates projected to the $\ell^{\text {th }}$ layer of turbulence, corresponding to the $i^{\text {th }}$ measurement in $z_{k}$, and the $j^{\text {th }}$ measurement in $s_{k}$. Here we do not discuss the specific functions $C_{\ell}$, only the fact that they depend on the projected subaperture coordinates and the system parameters $\rho$. These functions are well documented and an open-source implementation of Eqn. (4) can be found in the AOtools Python package, ${ }^{18}$ along with many other useful AO related functions.

\section{PREDICTION IN L\&A}

In order to improve the performance of the L\&A scheme, particularly with regard to frozen-flow ${ }^{19}$ turbulence, we provide a predictive step in the turbulence reconstruction. Specifically, to compensate for a pure-delay in the control loop of $d$ sampling periods, we propose estimating $z_{k+d}$ rather than $z_{k}$. This is achieved in the same way as in Eqn. (1), except with a modification of the time indices:

$$
\begin{aligned}
\hat{z}_{k} & =\left\langle z_{k+d} \mid s_{k}\right\rangle \\
& =\left\langle z_{k+d} s_{k}^{\top}\right\rangle\left\langle s_{k} s_{k}^{\top}\right\rangle^{-1} s_{k} .
\end{aligned}
$$

Notice that the matrices in Eqn. (5) have the same dimensions as those in Eqn. (1), showing that the extension from the non-predictive L\&A scheme to the predictive one comes at no increase in online computational burden.

To populate the time-shifted covariance matrix $\left\langle z_{k+d} s_{k}^{\top}\right\rangle$, we use Eqn. (4), except that we shift the coordinates by an amount equal to the distance they will be displaced due to frozen-flow evolution during a delay of $d \in \mathbb{R}$ sampling periods:

$$
\left\langle z_{k+d} s_{k}^{\top}\right\rangle_{i j}=\sum_{\ell=1}^{L} C_{\ell}\left(x_{i}^{\ell}+d T \nu_{x}^{\ell}, y_{i}^{\ell}+d T \nu_{y}^{\ell}, x_{j}^{\ell}, y_{j}^{\ell}, \rho\right),
$$

where $T$ is the sampling period and $\left(\nu_{x}^{\ell}, \nu_{y}^{\ell}\right)$ are the $(x, y)$ velocities of the $\ell^{\text {th }}$ layer of turbulence. 


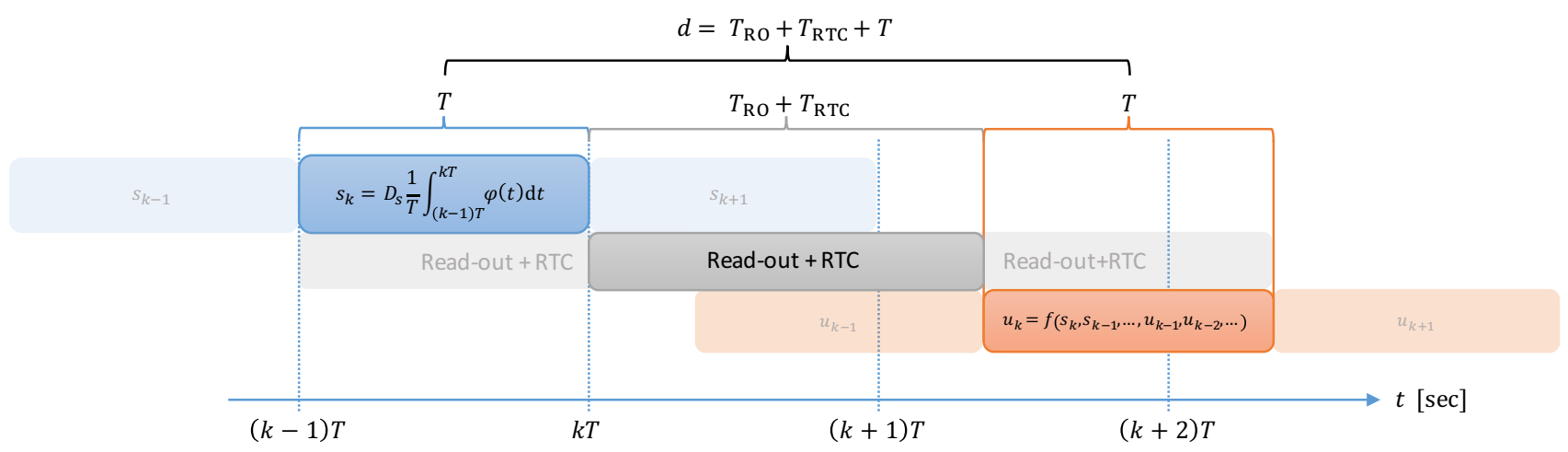

Figure 1. Timing diagram of AO loop including integration time, read-out time, and RTC time.

\subsection{Implementation in Closed-loop}

A non-integer value of the time delay, $d$, is handled automatically in the open-loop case, but for closed-loop systems special care is required to form the correct POLC scheme. This is demonstrated in the timing diagram shown in Figure 1. The pure-delay component is a result of the read-out time and RTC related computations/latency. We define the measurement $s_{k}$ as the one which is based on the integration over the time interval $t \in[(k-1) T, k T)$. This measurement is available in the RTC after $T_{\mathrm{RO}}$ seconds, and the command computed in the RTC is applied to the DM after an additional $T_{\mathrm{RTC}}$ seconds. We then assume that the DM shape is held constant over $t \in[(k+d-1) T,(k+d) T)$. We define $u_{k}$ as the command which is computed after receiving the measurement $s_{k}$ (despite the command not appearing at the DM until $t=(k+d-1) T$ ). This results in a closed-loop measurement in the general case given by:

$$
\begin{aligned}
s_{k}^{\mathrm{CL}} & =s_{k}-D_{s} \frac{1}{T} \int_{(k-d) T}^{(k-d+1) T} u(t) \mathrm{d} t \\
& =s_{k}-D_{s}\left(\Delta u_{k-\bar{d}}+(1-\Delta) u_{k-\bar{d}+1}\right) \\
\therefore s_{k} & =s_{k}^{\mathrm{CL}}+D_{s}\left((1-\Delta) u_{k-\bar{d}+1}+\Delta u_{k-\bar{d}}\right),
\end{aligned}
$$

where $D_{s}$ is the measurement interaction matrix, $u(t)$ is the instantaneous command signal (assuming negligible DM dynamics $), \Delta \triangleq(d \bmod 1)$, and $\bar{d} \triangleq \operatorname{ceil}(d)$.

We present for convenience the common case of two-frame delay $(d=2)$ :

$$
\begin{aligned}
s_{k}^{\mathrm{CL}} & =s_{k}-D_{s} u_{k-2} \\
\therefore s_{k} & =D_{s} u_{k-2}+s_{k}^{\mathrm{CL}} .
\end{aligned}
$$

To be convinced of this identity and to overcome the ambiguity of the modulo and rounding functions at integer values, note that the limit of Eqn. (8) as $d$ approaches 2 from $d<2$ is equal to the limit as $d$ approaches 2 from $d>2$.

The pure open-loop control law with no temporal filtering would be:

$$
u_{k}=R s_{k} .
$$

Embedding this into a first order unity-gain low-pass IIR filter gives:

$$
u_{k}=(1-g) u_{k-1}+g R s_{k}
$$

where the scalar $g \in(0,1)$ determines the cut-off frequency $f_{c}$ of the filter. This value can be tuned for a given system, or determined analytically from a desired value of $f_{c}$ :

$$
g=2-\cos \left(4 \pi f_{c} / f_{s}\right)-\sqrt{\left(2-\cos \left(4 \pi f_{c} / f_{s}\right)\right)^{2}-1},
$$


where $f_{c}$ and the control sampling frequency $f_{s}$ are both in the same units (e.g., Hertz).

Finally, we combine this first order open-loop IIR filter with Eqn. (9) to obtain the closed-loop control law:

$$
\begin{aligned}
u_{k} & =(1-g) u_{k-1}+g R\left(D_{s} u_{k-2}+s_{k}^{\mathrm{CL}}\right) \\
& =(1-g) u_{k-1}+g R D_{s} u_{k-2}+g R s_{k}^{\mathrm{CL}},
\end{aligned}
$$

or in the arbitrary delay case:

$$
\begin{aligned}
u_{k} & =(1-g) u_{k-1}+g R\left(s_{k}+D_{s}\left(\Delta u_{k-\bar{d}}+(1-\Delta) u_{k-\bar{d}+1}\right)\right) \\
& =(1-g) u_{k-1}+g(1-\Delta) R D_{s} u_{k-\bar{d}+1}+g \Delta R D_{s} u_{k-\bar{d}}+g R s_{k} .
\end{aligned}
$$

The first of these expressions is perhaps more appealing in the matrix notation. Eqn. (12) becomes:

$$
\left[\begin{array}{c}
u_{k} \\
u_{k-1}
\end{array}\right]=\left[\begin{array}{cc}
(1-g) & g R D_{s} \\
\mathbf{I} & \mathbf{0}
\end{array}\right]\left[\begin{array}{l}
u_{k-1} \\
u_{k-2}
\end{array}\right]+\left[\begin{array}{c}
g R \\
\mathbf{0}
\end{array}\right] s_{k}^{\mathrm{CL}} .
$$

Clearly an implementation in the matrix form without optimisation would be unnecessarily inefficient, but with some straightforward algorithmic modifications, the above (and also the general case of Eqn. (13)) can be performed with only 2 matrix-vector multiplications; one of dimensions $n_{\text {act }} \times n_{\text {act }}$ and the other $n_{\text {act }} \times n_{\text {meas }}$ (where $n_{\text {act }}$ is the number of actuators and $n_{\text {meas }}$ is the number of measurements).

\section{SIMULATIONS}

To evaluate the performance of the pL\&A reconstructor, we compare it with the classical L\&A implementation in the end-to-end numerical simulator, COMPASS. ${ }^{20,21}$ Both reconstructors are embedded in POLC with fixed $g=0.3$. The system parameters are chosen to be match where possible the expected MAVIS configuration for UT4 on the VLT. ${ }^{12}$ The simulation parameters are as follows:

- 8 LGS SH-WFS @ 17.5" radius (40×40 subapertures each),

- LGS: $6 \times 6$ pix, 5.39" FoV, 0.2 $\mathrm{e}^{-}$RON, 75 photon/sub-ap/ms flux @ 589nm,

- 3 NGS TT-WFS @ 20" radius $(1 \times 1$ subaperture each),

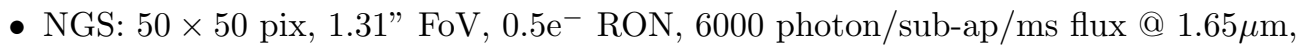

- 3 PZT DMs:

- $41 \times 41$ actuators, $0.2 \mathrm{~m}$ pitch @ 0km,

- $38 \times 38$ actuators, $0.25 \mathrm{~m}$ pitch @ 6km,

- $35 \times 35$ actuators, $0.32 \mathrm{~m}$ pitch @ 13.5km.

- 1ms WFS exposure time/control period,

- 2 frame delay (i.e., 1 frame pure-delay),

- 0.8" seeing @ zenith; $C_{n}^{2}$ profile in Table 1,

- $16 \%$ central obscuration,

- $8 \mathrm{~m}$ telescope diameter,

- 0.3 inter-actuator coupling factor,

- $30^{\circ}$ zenith angle,

- 30" diameter science FoV. 
Table 1. Cn2 Profile and Layer Velocities. Weights taken

\begin{tabular}{|c|c|c|c|}
\hline Altitude [m] & Weight & Effective $r_{0}$ & Velocity [m/s] \\
\hline 30 & 0.59 & 0.1623 & $6.6 \angle 0^{\circ}$ \\
140 & 0.02 & 1.2364 & $5.9 \angle 10^{\circ}$ \\
281 & 0.04 & 0.8157 & $5.1 \angle 20^{\circ}$ \\
562 & 0.06 & 0.6396 & $4.5 \angle 25^{\circ}$ \\
1125 & 0.01 & 1.8740 & $5.1 \angle 0^{\circ}$ \\
2250 & 0.05 & 0.7135 & $8.3 \angle 10^{\circ}$ \\
4500 & 0.09 & 0.5014 & $16.3 \angle 20^{\circ}$ \\
7750 & 0.04 & 0.8157 & $30.2 \angle 25^{\circ}$ \\
11000 & 0.05 & 0.7135 & $34.3 \angle 0^{\circ}$ \\
14000 & 0.05 & 0.7135 & $17.5 \angle 10^{\circ}$ \\
\hline
\end{tabular}

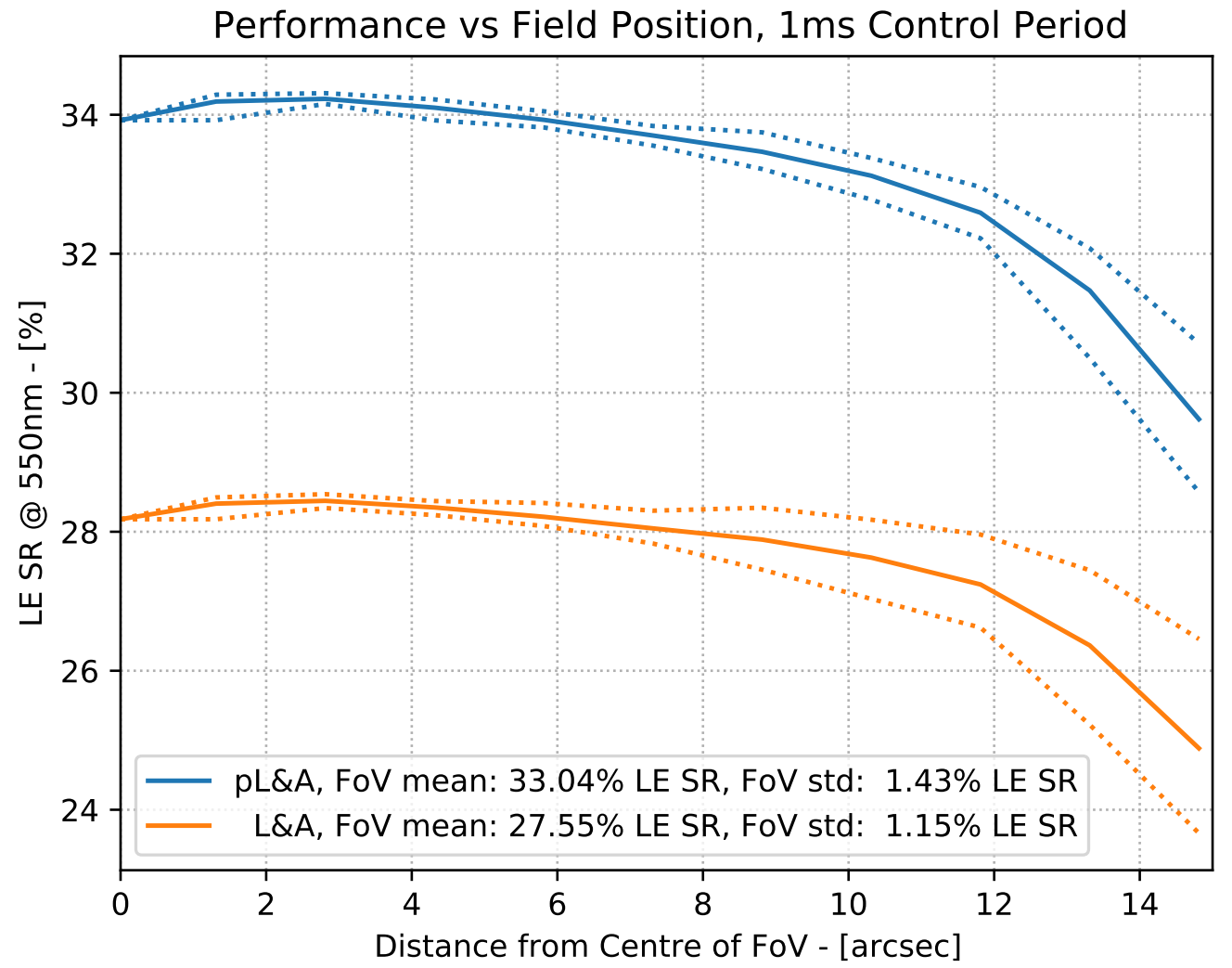

Figure 2. Predictive (blue) vs Non-Predictive (orange) Learn and Apply for $1 \mathrm{~ms}$ exposure time as a function of distance from the centre. Solid line is the mean, dotted lines are minimum and maximum.

Using these simulation parameters, the difference in performance of L\&A with and without prediction can be seen in Figure 2. The average long-exposure Strehl ratio (SR) over the science FoV is $33.04 \%$ and $27.55 \%$ for predictive and non-predictive L\&A respectively, corresponding to a improvement of $37.0 \mathrm{~nm}$ RMS in the MAVIS case under the conditions described above. Due to the tight error budget of MAVIS, ${ }^{12}$ this level of improvement is expected to have a considerable effect on the sky-coverage capabilities of the instrument.

Also of interest is the relative gain achieved by the predictive approach as the exposure time changes. Figure 3 demonstrates this as the exposure time changes from $0.5 \mathrm{~ms}$ to $2.5 \mathrm{~ms}$. It is clear that at very short exposure times, the gain is relatively small (approximately 20nm RMS at $0.5 \mathrm{~ms}$ exposure time). This is expected, due 


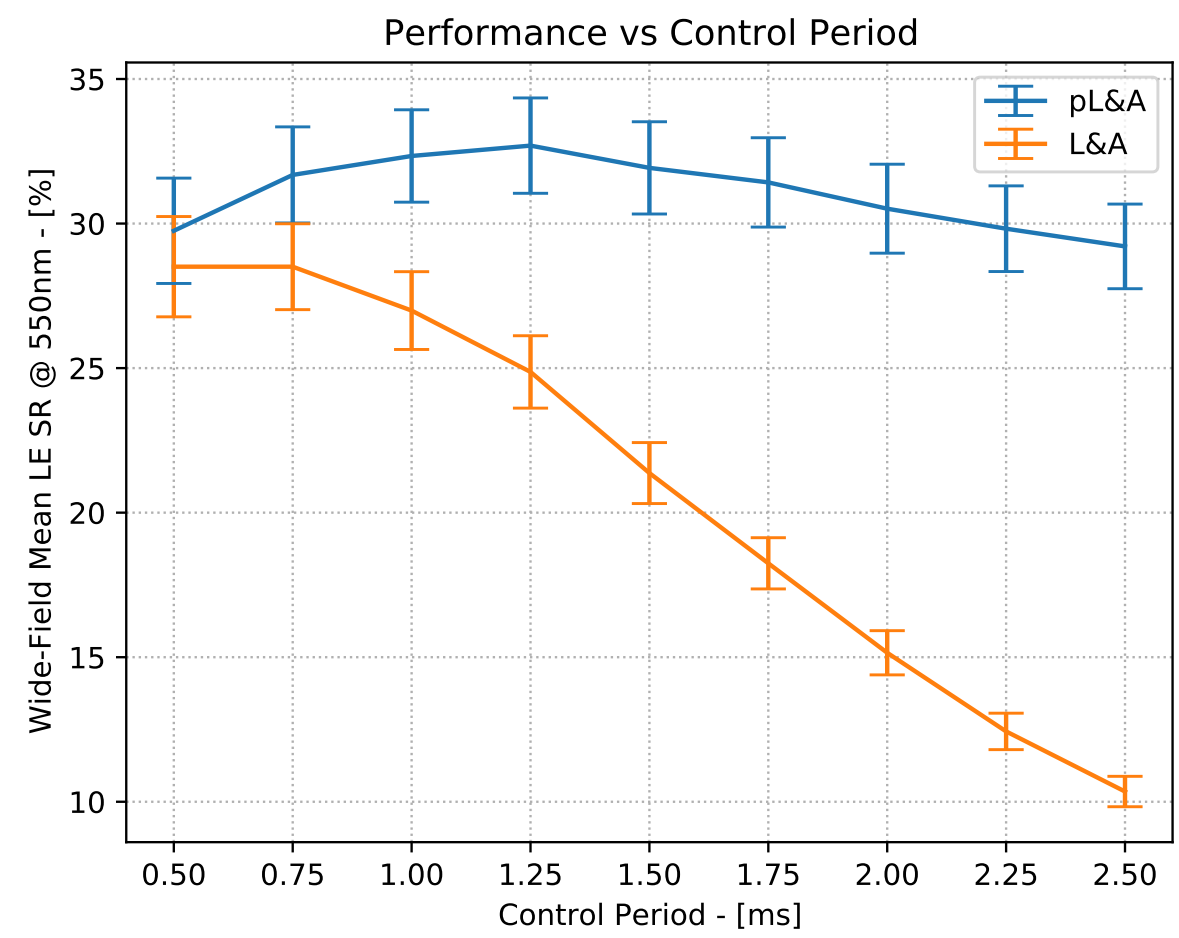

Figure 3. Predictive (green) vs Non-Predictive (blue) Learn and Apply for varying exposure time. Long Exposure SR averaged over the 30" diameter Science FoV.

to the temporal evolution of the turbulence at this time-scale being mostly negligible. As the exposure time increases, the performance of the non-predictive approach decays more rapidly than the predictive. The peak performance of the pL\&A based control scheme in this configuration occurs at $1.25 \mathrm{~ms}$ exposure time, where the improvement is $46 \mathrm{~nm}$ RMS across the FoV. The improvement becomes more significant at longer exposure times, as expected, with a difference of $88 \mathrm{~nm}$ RMS at $2.5 \mathrm{~ms}$.

Testing in existing simulation tools beyond this time-scale is not shown here, due to the decreasing validity of the frozen-flow assumption for longer time-scales.

\section{CONCLUSION}

Learn and Apply is a promising method for building MCAO tomographic reconstructors in systems such as MAVIS. The addition of a predictive step in the L\&A reconstructor provides an additional gain at no increase in online computational load. For the MAVIS case, end-to-end numerical simulations suggest an improvement of approximately $37 \mathrm{~nm}$ RMS wave-front error averaged over the science FoV at $1 \mathrm{~ms}$ exposure using pL\&A when compared to the non-predictive L\&A algorithm.

\section{REFERENCES}

[1] G. R. F. Vidal, E. Gendron, "Tomography Approach for Multi-Object Adaptive Optics," J. Opt. Soc. Am. A 27, No. 11 (2010).

[2] F. J. Rigaut, B. L. Ellerbroek, and R. Flicker, "Principles, Limitations, and Performance of Multiconjugate Adaptive Optics," in Adaptive Optical Systems Technology, P. L. Wizinowich, Ed., 4007, 1022 - 1031, International Society for Optics and Photonics, SPIE (2000).

[3] A. Tokovinin, M. Louarn, E. Viard, et al., "Optimized Modal Tomography in Adaptive Optics," A $\& A$ 378, $710-721$ (2001). 
[4] T. Fusco, J.-M. Conan, G. Rousset, et al., "Optimal Wave-Front Reconstruction Strategies for Multiconjugate Adaptive Optics," J. Opt. Soc. Am. A 18, 2527-2538 (2001).

[5] D. T. Gavel and D. M. Wiberg, "Towards Strehl-Optimizing Adaptive Optics Controllers," Proc. SPIE 4839 (2002).

[6] B. L. Roux, J.-M. Conan, C. Kulcsár, et al., "Optimal Control Law For Classical And Multiconjugate Adaptive Optics," J. Opt. Soc. Am. A 21, 1261-1276 (2004).

[7] C. Correia, K. Jackson, J.-P. Véran, et al., "Static And Predictive Tomographic Reconstruction For WideField Multi-Object Adaptive Optics Systems," J. Opt. Soc. Am. A 31, 101-113 (2014).

[8] C. M. Correia, K. Jackson, J.-P. Véran, et al., "Spatio-Angular Minimum-Variance Tomographic Controller for Multi-Object Adaptive-Optics Systems," Appl. Opt. 54, 5281-5290 (2015).

[9] H. Zhang, J. Cranney, N. Doucet, et al., "Predictive Learn and Apply: MAVIS Application - Learn," in (submitted to) Adaptive Optics Systems VII, International Society for Optics and Photonics, SPIE (2020).

[10] B. L. Ellerbroek and C. R. Vogel, "Simulations of Closed-Loop Wavefront Reconstruction for Multiconjugate Adaptive Optics on Giant Telescopes," in Astronomical Adaptive Optics Systems and Applications, 5169, 206 - 217, International Society for Optics and Photonics, SPIE (2003).

[11] P. Piatrou and L. Gilles, "Robustness Study of the Pseudo Open-Loop Controller for Multiconjugate Adaptive Optics," Appl. Opt. 44, 1003-1010 (2005).

[12] F. Rigaut, R. McDermid, G. Cresci, et al., "MAVIS Conceptual Design," in (submitted to) Ground-based and Airborne Instrumentation for Astronomy VIII, International Society for Optics and Photonics, SPIE (2020).

[13] G. Agapito, D. Vassallo, C. Plantet, et al., "MAVIS: System Modelling and Performance Prediction," in Adaptive Optics Systems VII, International Society for Optics and Photonics, SPIE (2020).

[14] T. Kailath, A. H. Sayed, and B. Hassibi, Linear Estimation, Prentice Hall (2000).

[15] Y. Hong, E. Bergou, H. Zhang, et al., "Stochastic Levenberg-Marquardt for Solving Optimization Problems on Hardware Accelerators," in (submitted to) 35th IEEE International Parallel \& Distributed Processing Symposium, IEEE (2021).

[16] M. Roggemann and B. Welsh, Imaging Through Turbulence, CRC Press (1996).

[17] E. Gendron, A. Charara, A. Abdelfattah, et al., "A Novel Fast and Accurate Pseudo-Analytical Simulation Approach for MOAO," Proc. SPIE 9148, 91486L-91486L-13 (2014).

[18] M. J. Townson, O. J. D. Farley, G. O. de Xivry, et al., "AOtools: a Python Package for Adaptive Optics Modelling and Analysis," Opt. Express 27, 31316-31329 (2019).

[19] L. Poyneer, M. van Dam, and J. P. Véran, "Experimental Verification of the Frozen Flow Atmospheric Turbulence Assumption With Use of Astronomical Adaptive Optics Telemetry," J. Opt. Soc. Am. A. 26(4) (2009).

[20] F. Ferreira, D. Gratadour, A. Sevin, et al., "Real-Time End-to-End AO Simulations at ELT Scale on Multiple GPUs with the COMPASS Platform," in Adaptive Optics Systems VI, 10703, 1155 - 1166, International Society for Optics and Photonics, SPIE (2018).

[21] F. Ferreira, D. Gratadour, A. Sevin, et al., "COMPASS: an Efficient GPU-Based Simulation Software for Adaptive Optics Systems," in 2018 International Conference on High Performance Computing Simulation (HPCS), 180-187 (2018). 